\title{
Deep Electrification of Residential Heating and Possible Implications: An Irish Perspective
}

\author{
Ankita Gaur*, Desta Fitiwi, and John Curtis \\ Economic and Social Research Institute and Trinity College Dublin, Ireland
}

\begin{abstract}
Electrifying energy sectors using renewable rich electricity is one of the many decarbonization pathways being adopted to curb greenhouse gas (GHG) emissions. Among these, the heating and cooling sector, both energy and carbon intensive, is attracting a lot of attention. Power-to-heat technology $(\mathrm{PtH})$ along with thermal energy storage systems is widely adopted to decarbonise this sector. However, increased penetration of $\mathrm{PtH}$ may cause congestion in existing electrical grid infrastructures, and hence needs for network upgrades. In this context, our paper presents a quantitative analysis on the impact of electrifying domestic dwellings (existing and new) in Ireland. The analysis encompasses costs, benefits, renewable power curtailment and regional distribution of optimal electrification of the housing stock. Analysis reveal significant grid expansion needs with increasing levels of PtH. This impact is pronounced without appropriate thermal storage. On the flip side, it leads to a more efficient utilisation of renewable energy by reducing curtailment.
\end{abstract}

\section{Nomenclature}

\section{Indices and Sets}

$g / \Omega^{g}$

$i, j / \Omega^{i}$

$k / \Omega^{k}$

$s / \Omega^{s}$

$w / \Omega^{w}$

$\Omega^{e \ell}, \Omega^{n \ell}$

$t / \Omega^{t}$

Index/set of all generators

Index/set of all nodes

Index/set of all lines

Index/set of scenarios

Index/set of operation states (e.g. hours)

Set of existing/new lines

Index/set of planning stages

$\begin{array}{ll}\text { Parameters } & \\ C^{H P} & \text { Capacity of heat pump } \\ C O P^{H P} & \text { Coefficient of performance of heat pump } \\ \alpha & \text { Losses in transmission network } \\ \rho_{s} & \text { Probability of a scenario } \\ \pi_{w} & \text { Weight of an operational state (hours) } \\ P D_{s, w, t}^{i} & \text { Active power demand (MW) } \\ r & \text { Interest rate (\%) }\end{array}$

Variables

$N_{s, w, t}^{r f, i}$

$P_{k, s, w, t}$

$P_{g, i, s, w, t}^{g e n}$

$P, P N S$

$P_{i, s, w, t}^{P N S}$

$P L_{k, s, w, t}$

$P_{s, w, t}^{H P, i}$

$P_{S, w, t}^{R F, i}$

$Q_{S, w, t}^{H P, i}$

$Q_{S, w, t}$

$Q_{s, w, t}^{n b, i}$
$Q_{s, w, t}^{r f, i}$
Functions
$E g y C_{t}^{g e n}$
$\operatorname{EmiC}_{t}^{g e n}$
$\operatorname{Inv} C_{t}^{n t k}$
$\operatorname{ENSC}_{t}$
$\operatorname{MntC}_{t}^{g e n}$
$\operatorname{MntC}_{t}^{n t k}$
$\operatorname{MntC}_{t}^{h p}$
$\operatorname{OpnC}_{t}^{h p}$

Heat demand in new builds (MJ)

Heat demand in existing houses (MJ)

\section{Introduction}

The residential sector is responsible for $25 \%$ of the energy consumed in Ireland, of which, space heating accounts for $61 \%$ to the total residential energy demand [1]. According to SEAI's 2018 report [1], space heating was responsible for $10 \mathrm{MtCO}_{2}$, which is about $17 \%$ of the total emissions in the Republic of Ireland (RoI). Presently, gas, oil and solid fuels are the major types of fuels being used for heating purpose in Irish homes, whereas electricity and renewables have negligible share. As part of the EU-wide decarbonisation drive, the RoI has set a renewable heat target of $12 \%$ by 2020 but is most likely to miss this. Yet, reducing emissions from the heating sector remains to be one of the key priorities of the country.

Among the possible decarbonisation pathways of this sector, the government's focus seems to have shifted to promoting the extensive adoption of heat pumps. There are 
policies intended to move this forward such as incentives for retrofitting existing houses. Heat pumps are generally energy efficient and environmentally friendly technologies that result in effective utilization and integration of intermittent renewable energy sources [2]. Analysis across several countries shows that heat pumps are superior to other alternatives since they reduce system costs, primary energy consumption and carbon emissions [3]-[5]. The study in [6] for instance demonstrates the capability of heat pumps in enabling higher renewable penetration and decreasing reserve capacity requirement. They not only enable low carbon heating but also increase the flexibility of a system. Various studies have shown that heat pumps can provide demand response, peak shaving, load shifting and energy conservation. [7],[8]. Heat pumps find extensive applications in district heating networks due to their potential of abating GHG emissions, supplying low cost heat, reducing wind power curtailment and, utilizing waste heat from data centres and combined heat and power (CHP) plants [9],[10].

The adoption of heat pumps along with thermal storage devices is proven to be more effective in utilizing excess renewable energy, increasing system flexibility and economic feasibility of heat pumps. Heat pumps can contribute to peak shaving, load shifting and energy conservation when combined with TES and hence reducing the additional peak load due to e-heating [11],[12]. During periods of high renewable generation, excess electricity can be converted to heat and stored in TES to be used later during peak heat demand period thereby reducing renewable curtailments.

According the Climate Action Plan 2019 for Ireland [13], the country has a target of achieving low carbon heat using renewables, biomethane and heat pumps. The detailed plan indicates of having 600,000 heat pumps all over Ireland by 2030, of which 200,000 are in new housing developments and 400,000 in old houses with retrofits. However, electrifying the heating sector at this scale or beyond may pose considerable challenges in the existing grid infrastructure. Hence, new network investments may be needed to fulfil this future electricity demand. In this paper, we present a quantitative analysis on the impacts of deep electrification on the electrical grid infrastructure, with a focus on the Irish case. For this purpose, we use a stochastic optimisation model [14], which is based on a linearised AC power flow.

\section{Algebraic Formulation}

The mathematical model consists of the transmission expansion planning (TEP) problem integrated with the residential heating sector. The objective function minimizes the overall system cost and is an extension of the one in [15] constrained with other techno-economic restrictions.

\subsection{Objective Function}

The objective function shown in (1) consists of seven cost terms, each associated with a certain relevance $\gamma_{m} ; \forall m \in\{1,2, \ldots, 7\}$. The cost terms are net present value (NPV) of investment cost
(TInvC) (2), maintenance (TMC) (3), operation (TEC) (4), load shedding (TENSC) (5), emissions (TEmiC) (6), operation and maintenance costs of heat pumps (HPC) (7) and cost of alternative heating system $(A H C)(8) . A H C$ roughly estimates the cost of serving the remaining balance of heat demand using alternate heating solutions (such as the conventional one). The PF parameter can therefore be understood as a proxy to the unit cost of alternative fuel and associated costs.

$$
\begin{array}{rl}
\text { Minimize TC }=\gamma_{1} & * \text { TInvC }+\gamma_{2} * T M C+\gamma_{3} * T E C \\
& +\gamma_{4} * \text { TENSC }+\gamma_{5} * \text { TEmiC }+\gamma_{6} \\
& * H P C+\gamma_{7} * A H C
\end{array}
$$

where,

$$
\begin{aligned}
& \operatorname{TInv} C=\sum_{t \in \Omega^{t}}(1+r)^{-t} \operatorname{Inv} C_{t}^{n t k} \\
& T M C=\sum_{t \in \Omega^{t}}(1+r)^{-t}\left(M n t C_{t}^{g e n}+M n t C_{t}^{n t k}\right) \\
& T E C=\sum_{t \in \Omega^{t}}(1+r)^{-t} E g y C_{t}^{g e n} \\
& T E N S C=\sum_{t \in \Omega^{t}}(1+r)^{-t} E N S C_{t} \\
& T E m i C=\sum_{t \in \Omega^{t}}(1+r)^{-t} \operatorname{EmiC}_{t}^{g e n} \\
& H P C=\mathrm{PF} * \sum_{t \in \Omega^{t}}(1+r)^{-t}\left(M n t C_{t}^{h p}+O p n C_{t}^{h p}\right) \\
& A H C=\sum_{t \epsilon \Omega} t(1+r)^{-t} \sum_{s \in \Omega^{s}} \rho_{s} \sum_{w \epsilon \Omega^{w}} \pi_{w}\left(Q_{s, w, t}^{r f, i}+\right. \\
& \left.Q_{s, w, t}^{n b, i}-Q_{s, w, t}^{H P, i}\right)
\end{aligned}
$$

\subsection{Electrical Network Systems Model}

The electrical network model is an extended version of that introduced in [16]. The active power balance equation is shown in (9). This equation includes the heat demand that is electrified via heat pumps. We assume that the reactive power remains unchanged by the introduction of heat pumps and is same as in [16].

$$
\begin{aligned}
\sum_{g \in \Omega^{g}} P_{g, i, s, w, t}^{g e n}+P_{i, s, w, t}^{P N S}+\sum_{i n, k \in \Omega^{k}} P_{k, s, w, t}-\sum_{\text {out }, k \in \Omega^{k}} P_{k, s, w, t} \\
=\sum_{\substack{i n, w \in \Omega^{w}\\
}} \frac{1}{2} P L_{l, s, w, t}+P D_{s, w, t}^{i} \\
+P_{s, w, t}^{H P, i} ; \quad k \in i ; g \in i
\end{aligned}
$$

A RES integration target shown in (10) is imposed. Other network-related constraints such as flow limits, network losses, power production limits and logical constraints which represent irreversibility of investment decisions are largely the same as those in [16].

$$
\begin{aligned}
& \sum_{s \in \Omega^{s}} \rho_{s} \sum_{w \epsilon \Omega^{w}} \pi_{w} \sum_{g \in \Omega^{R E S} \sum_{i \epsilon \Omega^{i}} P_{g, i, s, w, t}^{g e n}} \geq p_{t} * \\
& \sum_{s \in \Omega^{s}} \rho_{s} \sum_{w \epsilon \Omega^{w}} \pi_{w} \sum_{i \epsilon \Omega^{i}}\left(P D_{s, w, t}^{i}+H_{s, w, t}^{n b, i}+H_{s, w, t}^{r f, i}\right)
\end{aligned}
$$

\subsection{Residential Heating System Model}


First, the housing stock in the island is split into two parts: existing and new builds. The first category requires a shallow to deep retrofitting for e-heating to make economic sense, but new builds are assumed to be adequately insulated (and hence heat pump ready). Data for the latter category is provided by EirGrid. The 2016 census across the island is processed to obtain the number of houses connected to each electrical transmission node. Likewise, the heat demand is also aggregated to each power transmission node. This is done in accordance with electrical connectivity of each dwelling. In other words, the heat demand in each residential building is aggregated to the transmission node where the electricity comes from to the same building. The underlining assumption in this work is that all new builds use heat pumps. However, the number of old houses using heat pumps will depend on several aspects, one of which is the level of the penalty factor given in (11):

$$
Q_{s, w, t}^{n b, i} \leq Q_{s, w, t}^{H P, i} \leq\left(Q_{s, w, t}^{r f, i}+Q_{s, w, t}^{n b, i}\right)
$$

Equation (12) presents the total electricity demand of heat pumps at every node after considering a loss factor $(\alpha)$ in the heat demand due to heating network losses.

$P_{s, w, t}^{H P, i}(1-\alpha)=\left(Q_{s, w, t}^{H P, i}+Q_{s, w, t}^{c h, i}\right) / C O P^{H P}$

The number of houses electrified is defined by (13) as the maximum of the difference between the total heat demand and the heat demand from new builds divided by the capacity of a typical heat pump.

$N_{s, w, t}^{r f, i}=\left(Q_{s, w, t}^{H P, i}-Q_{s, w, t}^{n b, i}\right) / C O P^{H P}$

The thermal energy storage is modelled in a similar way as in [15]. We assume one thermal storage system installed at each dwelling, that is readied for e-heating.

\section{Data and Assumption}

We use the entire Irish power transmission system as a case study, considering its development out to 2030 . The input data consists of two parts: (i) hourly heat demand arising from new builds using heat pumps for domestic heating and (ii) the heat demand from old houses retrofitted by 2030 . The data related to the projected heating demand from new builds is provided by EirGrid [17]. Data on existing housing stock is taken from the Central Statistics Office (CSO), Ireland [18]. For the analysis in this work, an air source heat pump is chosen following an extensive literature survey [19]. The capacity of each heat pump is $1.504 \mathrm{~kW}$, in line with EirGrid's considerations. In addition to this, a thermal energy storage of $50 \mathrm{kWh}$ is assumed to be installed in each dwelling along with one heat pump.

The analysis considers two scenarios for nine different values of penalty factor. The "Direct" scenario represents e-heating with heat pumps only while the "With TES" scenario does e-heating with heat pumps and thermal energy storage systems.

\section{Results and Discussion}

Figure 1 shows the percentage of heat demand that is electrified via heat pumps for different PF values. In the "direct" scenario, changes in the penetration level of e-heating are not significant for a penalty factor setting of $80 € / \mathrm{MWh}$ or lower. Only beyond this threshold does the adoption of eheating make more economic sense. Whereas, when thermal storage systems are deployed as in the second scenario, the percentage of demand met via e-heating increases significantly from $40 € / \mathrm{MWh}$ onwards. This implies that it is far more economical to electrify residential heating when using TES as compared to the other case. Such a dramatic difference between the two scenarios is explained by the fact that the peak electrical and heating demands mostly happen during the same time periods. Hence, serving the heat demand becomes exorbitantly expensive in the "direct" scenario. But with TES, excess RES energy (that would otherwise be curtailed as in the "direct" scenario) is stored during periods of low heat and electrical demand and released during high demand and low RES power production periods.

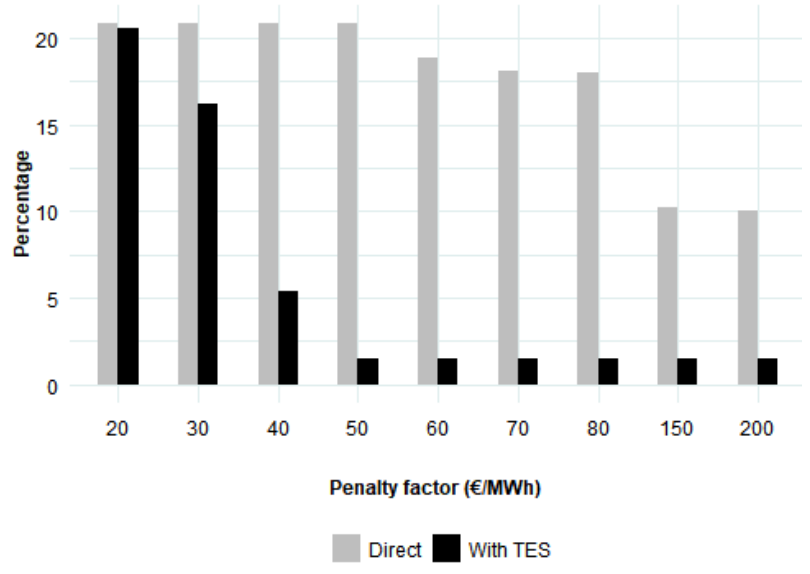

Fig.1. Optimal electrification level for various penalty factor values

Increasing level of e-heating also have profound impact on network investment needs. Figure 2 shows the additional transmission network capacity needed in both cases for various penalty factor settings. In the direct heating case, the expansion needs remains rather constant at $7.5 \%$ for $\mathrm{PF}$ values of $70 € / \mathrm{MWh}$ or lower. However, the higher the PF value is set

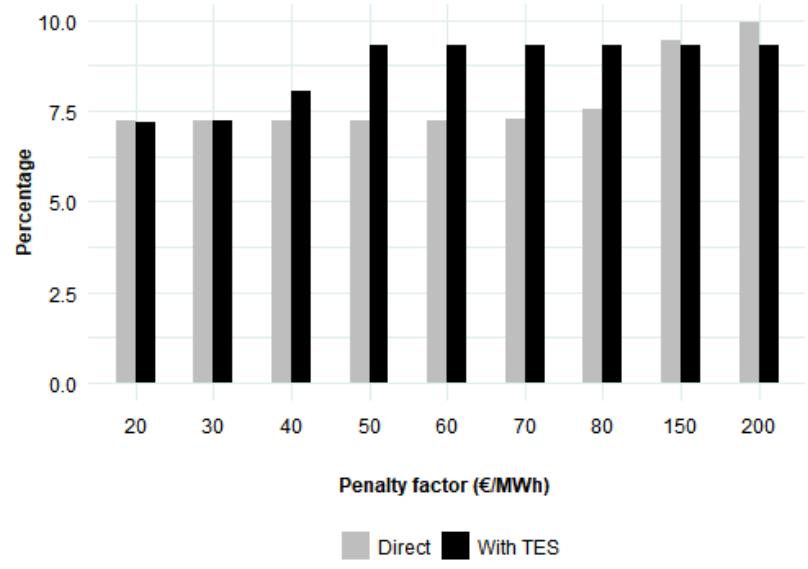

Fig. 2. Expected increase in network expansion needs (expressed as a percent of existing capacity) 
beyond $70 € / \mathrm{MWh}$, the higher the percentage increase in network expansion gets. This observation is in line with the optimal level of heat demand integration discussed earlier (see Figure 1).With TES, changes in grid expansion needs becomes significant beyond $30 € / \mathrm{MWh}$. This is due to the increased heat demand in this scenario as shown in Figure 1. At higher values of the penalty factor, the grid reinforcement needs is lower with TES, reflecting the benefits of installing heat pumps with TES.

Figure 3 compares the percentage of wind energy curtailed for both the scenarios. As can be seen, curtailment is substantially reduced with TES, leading to a more efficient utilization of available clean energy sources. A policy directed to incentivise the adoption of heat pumps should make sure that TES is co-integrated.

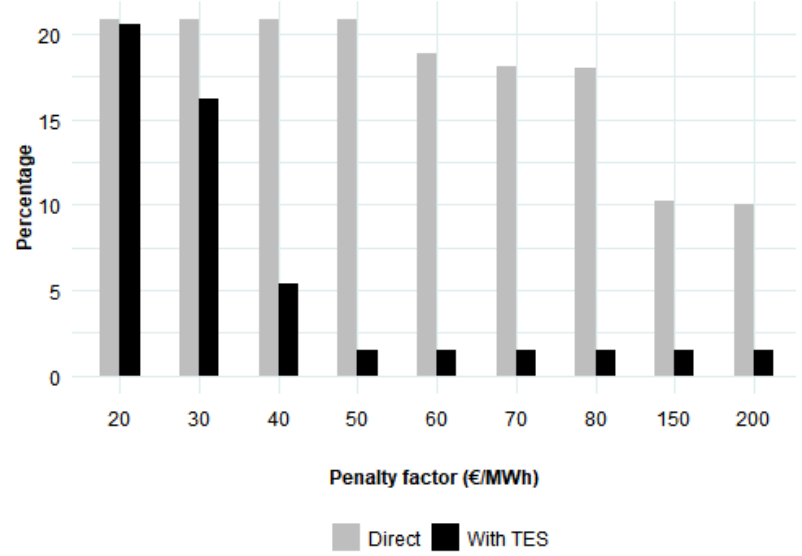

Fig. 3. Expected wind energy curtailment in both cases

The number of existing houses electrified in both scenarios is shown in Figure 4. In the scenario with TES, these numbers are in line with the heat demand profile (see Figure 1). However, in the direct scenario, there are literally no existing houses electrified until after the PF is set to $80 € / \mathrm{MWh}$. The electrified heat demand in this scenario is entirely due to new builds. Electrifying old houses becomes significant only for PF values of $150 € / \mathrm{MWh}$ or beyond. Even for a $\mathrm{PF}$ value of $200 € / \mathrm{MWh}$, more existing houses are electrified in the scenario with TES than in the direct one.

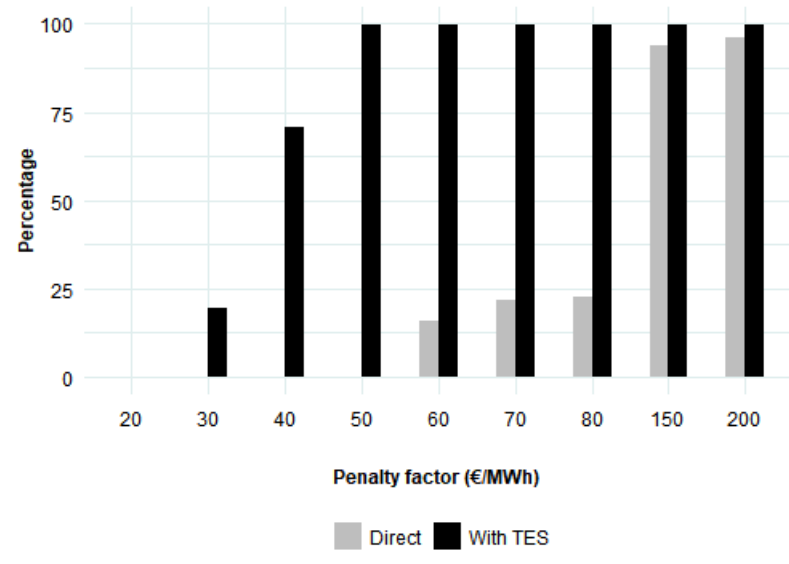

Fig. 4. Number of existing houses electrified
The regional distribution of optimally electrified houses is shown in Figure 5. The circles represent the percentage of houses electrified in each region. Note that the circles in each map are dimensioned based on the total number of houses electrified in each case. Hence, circles in one map should not be compared with those in any other map. In the direct scenario, for PF of $80 € / \mathrm{MWh}$, the small number of houses electrified are mostly located in the South-west region of Ireland, owing to the large wind energy potential in that region. Whereas, at $200 € / \mathrm{MWh} \mathrm{PF}$ value, most regions see modest increases in the number of houses electrified, but the highest electrification rates are in South-West, Dublin and surrounding areas.

With TES, a similar phenomenon is observed for lower PF settings (e.g. $30 € / \mathrm{MWh}$ ); more houses in the South-West are electrified due to high wind production potential in this region. For $80 € / \mathrm{MWh}$ PF setting, majority of the electrified houses lie in and around Dublin region, as well as in the South-West. It should be noted that the regional distribution of electrified houses is largely determined by the location of wind energy potential and network constraints. This is particularly relevant in the case of "direct" scenario. The results generally reveal that the co-deployment of TES leads to a substantially reduced network stress and enhanced system-wide flexibility, the combination of which enables a higher penetration level of eheating throughout the island.

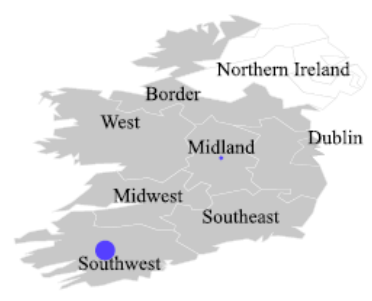

(i) $\mathrm{PF}=80 € / \mathrm{MWh}$; Direct

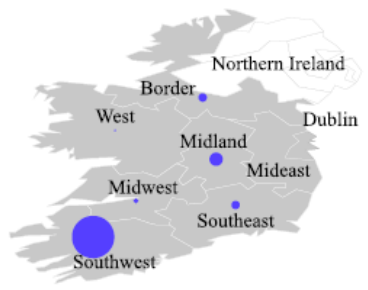

(iii) $\mathrm{PF}=30 € / \mathrm{MWh}$; With TES

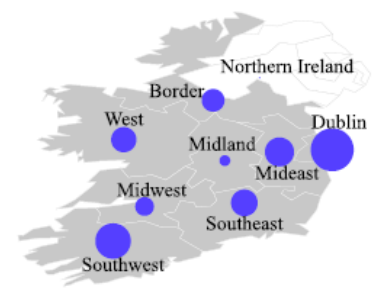

(ii) $\mathrm{PF}=200 € / \mathrm{MWh}$; Direct

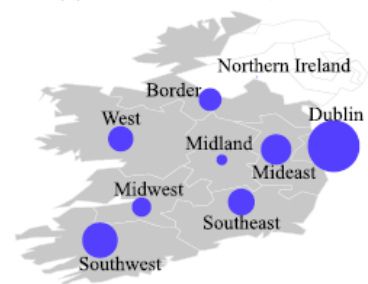

(iv) $\mathrm{PF}=80 € / \mathrm{MWh}$; With TES
Fig. 5. Regional distribution of optimally electrified houses

\section{Conclusion}

This paper has provided a quantitative analysis on the impact of deep electrification of the heating sector on the transmission infrastructure and other techno-economic aspects. The analysis determines the additional grid expansion needs by 2030 to supply the demand of increasing penetration of e-heating. We use the entire Irish power system to perform our analysis. The focus is on the impact of electrifying existing housing stock and new builds across the island on the grid expansion needs. We use a least-cost stochastic 
optimization model which is based on a linearised AC power flow to run case studies. Analysis results show that increasing the penalty factor-a proxy to the unit cost of meeting heat demand by alternative means other than e-heating - would increase the depth of heating sector electrification. This means that, for the considered system, as unit cost of alternate heating solution increases beyond ( $80 € / \mathrm{MWh}$ in the "direct" and 40 $€ / M W h$ in the "With TES" scenarios), it becomes economical to electrify the residential heating demand.

When the penetration of e-heating increases, the transmission grid expansion needs also increase. This is more pronounced in the "direct" case than in the "With TES" one. For the same level of electrification, grid expansion needs and emissions are lower in the latter case than in the former one. Electrifying the residential heating sector reduces renewable energy curtailment in the system particularly when deploying TES, thereby increasing the economic viability of investing in renewable technologies. Excess wind energy, during periods of low demand, is stored in TES and then released during the peak demand and low renewable power production periods. The co-deployment of TES increases the viability of electrifying existing housing stock in the island. In this regard, numerical results for the case study show that the number of old houses electrified are higher in the "With TES" case than in the "direct" one.

Generally, the higher the unit cost of alternative heating arrangement is, the more economical it becomes to electrify the residential heating sector. For the considered system, this threshold seems to be $80 € / \mathrm{MWh}$ and $40 € / \mathrm{MWh}$ in the "direct" and the "With TES" scenarios, respectively. TES enhances the performance of heat pumps and overall system flexibility, thereby making them advantageous for system operation. The regional distribution of existing houses electrified is determined by the location of renewable sources and network constraints. Given the limitations of the existing electrical network system, it is deemed economical to electrify more houses located near RES-rich places. This is particularly the case when TES is not co-deployed with heat pumps.

\section{ACKOWLEDGEMENT}

This work was supported by Science Foundation Ireland (SFI) under the SFI Strategic Partnership Programme [grant number SFI/15/SPP/E3125]; and the Economic and Social Research Institute's Energy Policy Research Centre. The opinions, findings and conclusions or recommendations expressed in this material are those of the authors and do not necessarily reflect the views of the Science Foundation Ireland..

This work has benefited from the valuable support of EirGrid, the Transmission System Operator in the Republic of Ireland.

\section{REFERENCES}

[1] "Energy in the residential sector," Sustainable Energy Authority of Ireland, Tech. Rep., 2018. [Online]. Available: https://www.seai.ie/resources/seaistatistics/keypublications/en ergy in-residential-sector/

[2] W. Yunna and X. Ruhang, "Green building development in China-based on heat pump demonstration projects," Renewable Energy, vol. 53, pp.211-219, May 2013.

[3] S. A. Alla, V. Bianco, A. Marchitto, F. Scarpa, and L. A. Tagliafico, "Impact of the Utilization of Heat Pumps for Buildings Heating in the Italian Power Market," in 2018 15th International Conference on the European Energy Market (EEM). Lodz: IEEE, Jun. 2018, pp. 1-5.

[4] S. N. Petrovi'c and K. B. Karlsson, "Residential heat pumps in the future Danish energy system," Energy, vol. 114, pp. 787797, Nov. 2016.

[5] A. M. Brockway and P. Delforge, "Emissions reduction potential from electric heat pumps in California homes," The Electricity Journal, vol. 31, no. 9, pp. 44-53, Nov. 2018.

[6] K. Hedegaard and M. M"unster, "Influence of individualheat pumps on wind power integration - Energy system investments and operation," Energy Conversion and Management, vol. 75, pp. 673-684, Nov. 2013.

[7] J. Love, A. Z. Smith, S. Watson, E. Oikonomou, A. Summerfield, C. Gleeson, P. Biddulph, L. F. Chiu, J. Wingfield, C. Martin, A. Stone, and R. Lowe, "The addition of heat pump electricity load profiles to GB electricity demand: Evidence from a heat pump field trial," Applied Energy, vol. 204, pp. 332-342, Oct. 2017.

[8] A. D. Carvalho, P. Moura, G. C. Vaz, and A. T. de Almeida, "Ground source heat pumps as high efficient solutions for building space conditioning and for integration in smart grids," Energy Conversion and Management, vol. 103, pp. 991-1007, Oct. 2015.

[9] M. K"ofinger, D. Basciotti, R. Schmidt, E. Meissner, C. Doczekal, and A. Giovannini, "Low temperature district heating in Austria: Energetic, ecologic and economic comparison of four case studies," Energy, vol. 110, pp. 95-104, Sep. 2016.

[10] F. Levihn, "CHP and heat pumps to balance renewable power production: Lessons from the district heating network in Stockholm," Energy, vol. 137, pp. 670-678, Oct. 2017.

[11] M. Pau, J. L. Cremer, F. Ponci, and A. Monti, "Impact of customers flexibility in heat pumps scheduling for demand side management," in 2017 IEEE International Conference on Environment and Electrical Engineering and 2017 IEEE Industrial and Commercial Power Systems Europe (EEEIC / I\&CPS Europe). Milan, Italy: IEEE, Jun. 2017, pp. 1-6.

[12] A. Arteconi and F. Polonara, "Assessing the Demand Side Management Potential and the Energy Flexibility of Heat Pumps in Buildings," Energies, vol. 11, no. 7, p. 1846, Jul. 2018.

[13] "Climate action plan 2019," Government of Ireland, Tech. Rep., 2019. [Online]. Available: https://www.dccae.gov.ie/enie/climateaction/ topics/climate-action-plan/Pages/climateaction.aspx

[14] D. Z. Fitiwi, M. Lnych, and V. Bertsch, "Optimal development of electricity generation mix considering fossil fuel phase-out and strategic multi-area interconnection," ESRI Working Paper, Tech. Rep., 2019.

[15] A. Singh Gaur, D. Fitiwi, and J. Curtis, "Implications of Powerto-Heat on Transmission Expansion Needs: A Real Life Case Study," in 2019 13th IEEE PowerTech Milano. IEEE, Jun. 2019, pp. 1-6.

[16] D. Z. Fitiwi and V. Bertsch, "Interactions between Demand Response and Network Expansion Planning: A Quantitative Analysis," in 2018 15th International Conference on the European Energy Market (EEM), Jun. 2018, pp. 1-6. 
[17] EirGrid, "Tomorrows-Energy-Scenarios-Report-2017," EirGrid, Tech. Rep., Jul. 2017.

[18] Central Statistics Office, "Census 2016 Small Area Population Statistics," 2016. [Online]. Available: https://www.cso.ie/en/census/census2016reports/
[19] A. Singh Gaur, D. Fitiwi, and J. Curtis, "Heat pumps and their role in decarbonising heating sector: a comprehensive review," ESRI Working Paper, Tech. Rep., 2019. 